\title{
Pseudoconditioning as a function of specific schedules of interstimulus intervals
}

\author{
BARRY LEDWIDGE and KENNETH R. BURSTEIN \\ Simon Fraser University, Burnaby, British Columbia, Canada V5A 1S6
}

\begin{abstract}
Two groups were presented with pseudoconditioning procedures identical with respect to the mean and standard deviation of the interstimulus intervals involved, but differing with respect to the proportion of interstimulus intervals occurring at particular ranges. Confirming and extending the results of Boneau (1958) and of Prokasy et al. (1963), differences in modal interstimulus intervals resulted in correlated changes in mean response latency. These findings are interpreted as supporting the view that the difference between responses obtained with conditioning and pseudoconditioning procedures is quantitative rather than qualitative.
\end{abstract}

Although the phenomenon of pseudoconditioning (PC) was demonstrated by Grether as early as 1938, the similarity of the operations for obtaining PC and for obtaining classical conditioning did not lead to the immediate use of PC control groups in classical conditioning experiments. It was not until Mowrer and Aiken (1954) introduced a control group in their study of the acquisition of fear responses that an attempt was made to include safeguards in conditioning procedures against the possible artifactual effects of $\mathrm{PC}$ and sensitization.

Since then, the specific procedures employed in PC control groups have been increasingly questioned and criticized on both logical and theoretical grounds. In a theoretical paper Rescorla (1967), after examining and finding inadequate each of the control procedures typically employed, argued convincingly for a truly random procedure as the appropriate control for classical conditioning.

More recently, Burstein (1973) reviewed the various procedures included under the rubric of classical conditioning (classical conditioning with partial reinforcement, temporal conditioning, and classical conditioning in which the interstimulus interval (ISI) is systematically shifted) and concluded that conditioning and PC procedures differ only in terms of the degree of ISI variability, with zero ISI variability, at one end of the dimension, constituting classical conditioning and completely random ISI variability, at the other end of the dimension, constituting PC.

As noted by Kling and Riggs (1972, p. 577) and Kimble (1961, p. 63), PC and sensitization are typically viewed as involving nonassociative processes. Burstein's view, that $\mathrm{PC}$ and classical conditioning differ only with respect to the degree of ISI variability, explicitly assumes that the processes underlying these two

This research was supported by Grant APA-193 from the National Research Council to the junior author. phenomena are identical, but that different degrees of ISI variability result in different levels of responding, particularly with the procedures conventionally employed for response definition.

One of the implications of this position is that, if ISI variability is manipulated, significant differences in responding can be obtained between two PC groups, although it is quite unlikely that a different process is operating in each of these two PC groups. If such differences can be obtained between two PC groups in which different underlying processes are clearly not involved, it would seem questionable to assume that similar differences obtained between PC and classically conditioned groups reflect a difference in underlying processes.

Burstein's view leads to the prediction that the frequency and latency of responding, in both PC and classical conditioning, is a function of such parameters as the average duration of the ISI, the degree of variability of the ISI, the specific ISI schedule, and the proportion of CSs that are followed by UCSs. This prediction is based partially upon the findings of Boneau (1958) and Prokasy and his associates that increases and decreases in the ISI resulted in correlated changes in mean response latencies. Ebel and Prokasy (1963) shifted ISIs, in an increasing and decreasing fashion, between $.2, .5$ and $.8 \mathrm{sec}$, and reported that mean response latency was related to changes in the ISI, a finding which confirmed and expanded the results of Boneau (1958). Prokasy, Ebel, and Thompson (1963) extended this range of ISIs and reported similar results for ISIs ranging from .630 to $2.497 \mathrm{sec}$. Though Prokasy and his associates employed blocks of trials at a one particular ISI before shifting to blocks of trials at another ISI, it is noteworthy that the PC procedure can be viewed as one in which the $\mathrm{N}$ of each "block" of trials has been reduced to 1 .

This study was designed to test the hypotheses that, in PC procedures, the frequency and the latency of responding is a function of the modal ISI. 

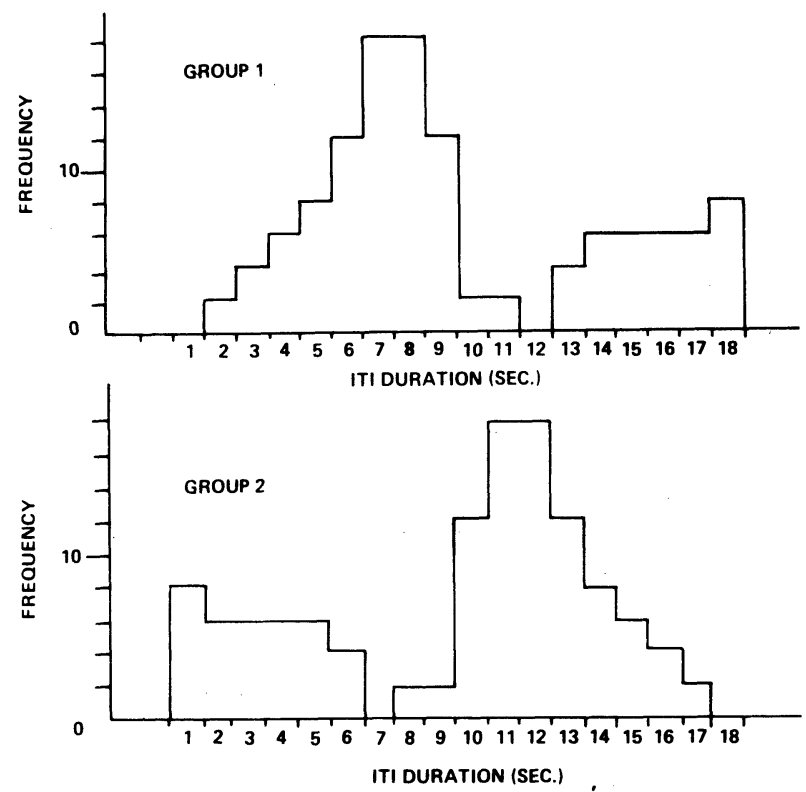

Figure 1. Frequency distributions of ITIs in each group.

\section{METHOD}

\section{Subjects}

The subjects were 30 university students who responded to appeals for paid $(\$ 1)$ volunteers to serve as subjects in an experiment on stress. They were alternately assigned to one of the two PC groups of equal size.

\section{Apparatus}

The subject was seated in a comfortable armchair in a $6 \times 6 \mathrm{ft}$ IAC soundproof booth. The experimenter could see the subject through a oneway screen and hear the subject through an intercom.

The CS was a .5-sec 1000-cps tone delivered through earphones by a Marconi wide-range oscillator (Model TF2103) at a sound intensity of $60 \mathrm{db}$.

The UCS consisted of a .1-sec shock, at a level previously determined by the subject to be unpleasant but not painful, delivered to the left wrist. The electrode used to deliver the shock was the concentric shock electrode developed by Tursky, Watson, and O'Connell (1965).

The skin conductance was measured continuously using a Fels dermohmeter and recorded by an Esterline-Angus rectigraph. The GSR recording system consisted of zinc electrodes, $7 / 8$ in. in diam, in a plastic cup filled with zinc-sulphate electrolyte.

The stimuli was presented automatically by a Tally tape reader and stimulus duration was controlled by Grason-Stadler timers (Model E1100H). A computer program was written which translated the schedule for each subject into a punched tape. Thirty computer tapes were thus generated, one for each subject. The tape reader was driven by electrical pulses $.5 \mathrm{sec}$ apart which were timed by a Grason-Stadler 1180 electronic timer.

\section{Procedure}

When the subject arrived for the session he was seated in the chair and was told that he would hear some tones through earphones, that he would feel some shocks from an electrode on his wrist, and that recordings would be made from electrodes on his palms.

He was assured that the shock he would receive would be at a level determined by himself to be unpleasant but not painful. He was advised that he could leave at this point, or at any time during the session.
The GSR and shock electrodes were then affixed in place; a level shock determined by the subject to be unpleasant but not painful was determined by stepping up the shock in increments of $.5 \mathrm{~mA}$ until the subject felt something and in increments of $.1 \mathrm{~mA}$ until the subject reported that it was unpleasant.

The subject was asked to try to keep still during the session so as not to disturb the recording apparatus, the earphones were placed on his head, the door to the soundproof room was closed, and the session began.

The pseudoconditioning was preceded by adaptation to the tone, $10 \mathrm{CS}$-alone presentations, the intervals between which varied from 11 to $28 \mathrm{sec}$.

The $120 \mathrm{CSs}$ and UCSs which followed the adaptation phases were presented randomly and were different for each subject with three constraints: (1) Every stimulus sequence consisted of exactly $60 \mathrm{Cs}$ and 60 UCSs; (2) the ISI interval following every sixth CS was drawn from among those intervals equal to or greater than $14 \mathrm{sec}$ in order to insure that every sixth trial could serve as a test trial; (3) though the specific sequence of ISIs differed for each subject, the summary distribution was identical for each subject in the same group. As can be seen in Figure 1, the two summary distributions mirrored each other. The groups differed only in that $40 \%$ of the ISIs in Group 1 were 6-8 sec inclusively, while $40 \%$ of the ISIs in Group 2 were 10-12 sec inclusively. The remainder of the ISIs in each group were distributed in such a way that the mean and the standard deviations of the two groups were identical $(9.50 \mathrm{sec}$ and $4.54 \mathrm{sec}$, respectively).

\section{Scoring}

A GSR was defined as any measurable deflection of the pen in the direction of increased conductance. Although the hypothesis being tested generates a prediction only with respect to relative responding at the latencies appropriate to the modal ISIs of the two groups, all responses in the 1-16-sec interval following test CSs were scored so that the entire latency distribution could be examined.

Responses appropriate to the modal intervals of the two groups were defined as responses occurring between 7 and 11 sec and between 11 and $15 \mathrm{sec}$, respectively. The lower limit of the scoring interval for responses appropriate to the modal ITI was set at the lower limit of the modal ITI range plus $1 \mathrm{sec}$, since no galvanic skin response is physiologically possible with a latency of less than $1 \mathrm{sec}$. The upper limit of the scoring interval for responses appropriate to the modal ITI was restricted to the upper limit of the modal ITI range plus $2 \mathrm{sec}$, since the latency range of the GSR has been found to fall between 1.0 and $2.9 \mathrm{sec}$ (McDonald and Johnson, 1965; Burstein and Epstein, 1967).

Using a specially devised scoring key, responses following every sixth CS were scored. The scoring key divided the ISI following the $6 \mathrm{th}, 12 \mathrm{th}, 18 \mathrm{th}, 24 \mathrm{th}, 30 \mathrm{th}, 36 \mathrm{th}, 42 \mathrm{nd}, 48 \mathrm{th}$, 54 th and 60 th CSs into 161 -sec intervals. The occurrence or nonoccurrence of a response in each of the 1-sec intervals was noted.

\section{RESULTS}

\section{Galvanic Skin Response Data}

The distribution of the latencies of all galvanic skin responses in the 16-sec scoring interval following every 6th CS (Figure 2) reveals that the latency range producing the most responses is the OR latency range (i.e., greater than $1 \mathrm{sec}$ but less than $4 \mathrm{sec}$ ).

Further examination of the distribution of the latencies of all responses reveals that beyond the OR latency range more responses in each group occur within the modal intertrial interval than elsewhere, incorporating an appropriate delay for the typical latency of the 


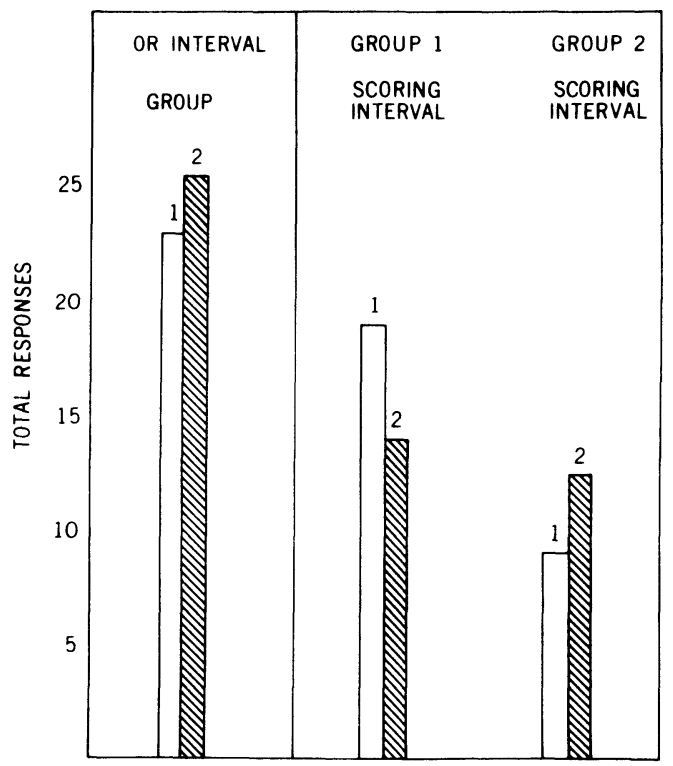

Figure 2. Distribution of GSR latencies for each group.

galvanic skin response as per the scoring procedure outlined previously.

An analysis of variance was performed on the frequency of responding in the designated scoring intervals. Scoring Interval 1 is from 7 to $11 \mathrm{sec}$; Scoring Interval 2 is from 11 to $15 \mathrm{sec}$. The data consist of 600 scores ( 2 groups by 15 subjects by 10 test trials by 2 scoring intervals). Each score represents the number of GSRs that occurred in that subject-trial-interval.

The hypothesis that responding at the latency of the modal ITI in each group would be greater than at the latency of the modal ITI of the other group is equivalent to predicting a significant Groups by Scoring Interval ( $G$ by SI) interaction in the analysis of variance.

The results of the analysis are presented in Table 1. All Fs are nonsignificant with the exception of the $G$ by SI interaction $(\mathrm{F}=6.79, \mathrm{df}=1.28, \mathrm{p}<.025$ or $\mathrm{p}<.0125$ for the one-tailed hypothesis tested).

The nonsignificant Group by Trial by Interval (GTI) interaction $(F=1.154, \quad d f=9 / 252)$ allows an unambiguious interpretation of the results. The significant $G$ by $I$ interaction indicates that the relative responding in the two scoring intervals is a function of which group the subject is in, and the nonsignificant GTI higher order interaction uncomplicates the picture since $\mathrm{G}$ by $\mathrm{I}$ interaction is not, then, a function of the particular trial.

\section{DISCUSSION}

These results, therefore, would seem to support the findings of Prokasy and his associates that changes in CS-
UCS intervals result in correlated changes in response latency. Moreover, they extend the validity of Prokasy's findings to apply to procedures which are not typically regarded as associational, since they demonstrate that highly significant differences can be obtained between pseudoconditioned groups even though the means and standard deviations are identical in these groups.

The present findings may be relevant to recant controversies as to what is the proper control procedure for demonstrating classical conditioning (Rescorla, 1967), and as to whether there can ever be an appropriate control procedure for eliminating the effects of orienting responses and pseudoconditioned responses, for they add weight to the view that the differences between the performances of classically conditioned groups and control groups is a quantitative one, rather than a qualitative one.

\section{REFERENCES}

Boneau, C. A. The interstimulus interval and the latency of the conditioned eyelid response. Journal of Experimental Psychology, 1958, 56, 464-472.

Burstein, K. R. On the distinction between conditioning and pseudoconditioning. Psychophysiology, 1973, 10, 61-66.

Burstein. K. R., \& Epstein, S. Primary stimulus generalization as a function of objective and subjective definition of the stimulus dimension. Journal of Experimental Psychology, 1967, 74, 124-131.

Ebel, H. C., \& Prokasy, W. F. Classical eyelid conditioning as a function of sustained and shifted interstimulus intervals. Journal of Experimental Psychology, 1963, 65, 52-58.

GRETHER, W. F. Pseudo-conditioning without paired stimulation encountered in attempted backward conditioning. Journal of Comparative Psychology, 1938, 25, 91-96.

Kling, J. W. \& Riggs, L. A. Woodworth and Schlosberg's Experimental Psychology. (3rd ed.) Vol. II. New York: Holt, Rinehart \& Winston, 1972.

Kimble, G. A. Hilgard and Marquis' conditioning and learning. (2nd ed.) New York: Appleton, 1961.

McDonald, D. G. \& Johnson, L. C. A re-analysis of GSR conditioning. Psychophysiology, 1965, 1, 291-295.

Mowrer, O. H. \& AIKEN, E. G. Contiguity vs. drive-reduction in conditioned fear. American Journal of Psychology, 1954, 67, 26-38.

Prokasy, W. F. Ebel, H. C., \& Thompson, D. D. Response shaping at long interstimulus intervals in classical eyelid conditioning. Journal of Experimental Psychology, 1963. 66, 138-141.

Rescorla, R. A. Pavlovian conditioning and its proper control procedures. Psychological Revien, 1967, 74, 71-80.

Tursky, B., Watson, P. D., \& O'Connell, D. N. A concentric shock electrode for pain stimulation. Psychophysiology, 1965. 1. 296-298. 